\title{
TEMAS CONTROVERTIDOS EN EDUCACIÓN CIENTÍFICA
}

\author{
Anna María Pessoa de Carvalho e Andéa Infantosi Vannucchi \\ Faculdade de Educaçao. Universidade de São Paulo \\ Av. da universidede 308; 05508-900, São Paulo, Brazil
}

\begin{abstract}
This article discusses and presents data showing the need and potentiality of controversial themes to science education, in contrast to dogmatic content traditionally constitutive of such courses. It is argued that the discussion about science through a historical approach can oppose the latter. Never the less, it is necessary that these contents be not introduced in the science classroom as a "metoric of conclusions". It is thus analyzed, through data obtained by videotape recording, how students work on an activity designed according to both content and methodological precepts, having as focus the relationship between science and technology and historically set on the improvement of the spyglass by Galileo in the I7th century.
\end{abstract}

\section{RESUMEN}

Este artículo discute y presenta datos que plantean la necesidad y el potencial de temas controvertidos para la educación científica, por oposición al contenido dogmático que la Ciencia representa en la enseñanza tradicional, lo cual puede ser factible mediante la discusión sobre Ciencia dentro de su contexto histórico. Sin embargo, es necesario que dicho contenido no se introduzca en el aula como una "retórica de conclusiones". A través de datos obtenidos por grabación en video, se analiza el modo como los alumnos discuten una actividad elaborada según tales preceptos, enfocada hacia las relaciones entre Ciencia y Tecnología, históricamente ubicada en el episodio de perfeccionamiento de la luneta por Galileo Galilei en el siglo XVII.

\section{INTRODUCCIÓN}

Una queja común entre los profesores de Física es que sus alumnos buscan, siempre, respuestas listas y algoritmos por medio de los cuales puedan resolver los problemas que se les plantean en las clases. Esa queja suele ocurrir también entre los profesores de estos profesores, ya que los profesores-alumnos buscan, y a veces lo solicitan directamente, un manual que les indique, a lo mejor por 'rubros ordenados, la manera correcta de dar una buena clase.

En el primer caso, se podría calificar la solicitud de los alumnos como improcedente con el siguiente argumento: no existe un algoritmo o manual de cómo hacer Ciencia o abordar problemas científicos. En el caso de los profesores-alumnos la respuesta seria análoga: no existe una manera correcta de dar clases y, por lo tanto, no puede haber un manual sobre cómo dar una buena clase - con un agregado: ¿qué caracteriza una buena clase? 
En este artículo se argumentará que la razón para tal solicitud que hacen profesores y alumnos está condicionada a por lo menos dos motivos en común: el contenido y la forma de educación escolar a que ambos grupos estuvieron o están sometidos. Se ha constatado que:

1. La Ciencia escolar es dogmática (Carvalho \& Gil-Pérez, 1995), y se presenta como conjunto de verdades establecidas a través del "Método Científico", que permite extraerlas de la naturaleza - ya en el siglo XVII, están las palabras de Bacon: [....] Y lo que se presenta como causa en la contemplación, es la regla en la práctica" (1973, aforismo III, tomo 1).

Y, asociada a este enfoque del contenido,

2. La organización escolar es autoritaria, o bien, al otro extremo, se aproxima al laissez-Faire (Ausubel, 1978; Hodson, 1987, apud Carvalho \& Gil-Pérez, 1995). La acción del profesor, en detrimento de un discurso crítico-liberal es, muchas veces, dogmática y represiva (Carvalho, 1989).

Se presentará el enfoque histórico-filosófico como alternativa para la visión rígida y algorítmica representada por la Ciencia escolar. Sin embargo, dicho enfoque es condición necesaria más no suficiente. Debe haber congruencia entre el contenido y la metodología de trabajo en clase - y el contexto de la clase, por lo general, no suele fomentar la reflexión, la argumentación y la comunicación de ideas (Trumbull, 1987).

Se analizarán datos obtenidos en clase, cuando se grabó en vídeo y se transcribió la discusión de una actividad que enfocaba ambos aspectos - de contenido y forma. Su análisis muestra la necesidad y el potencial de temas controvertidos en la educación científica, por lo que concierne a la comprensión de la Ciencia como visión del mundo y la actividad científica como proceso permanente de construcción de conocimiento.

\section{CONTENIDO Y FORMA: CONGRUENCIA}

A continuación se analizan el contenido y la metodología que suele adoptar en el aula el profesor de materias científicas. La Historia y la Filosofía de la Ciencia se plantean como un enfoque que se puede contraponer a la visión de sentido común, siempre y cuando el trabajo en clase sea coherente con tal perspectiva.

Aunque las cuestiones de contenido y metodología se analicen aparte, en diversos momentos se observan proyecciones y superposiciones mútuas, porque al fin de cuentas no se trata de aspectos perpendiculares.

\subsection{Sobre el contenido}

Diversas investigaciones han mostrado la visión de alumnos y profesores de las Ciencias sobre el conocimiento y la metodología científica (por ejemplo, Gil-Pérez, 1993; Hodson, 1993; Meichstry, 1993; Guilbert \& Meloche, 1993, apud Gil-Pérez, 1995 y, en Brasil, Moraes et al., 1990).

Según tales profesores, la Ciencia se caracteriza como un conjunto de verdades absolutas e inmutables. Coherentemente, en sus clases la presentan en su estado "final", 
sin ninguna referencia ya sea a situaciones problemáticas relativas a su origen 0 evolución histórica, ya sea a las limitaciones de ese conocimiento (Gil-Pérez, 1995).

En contraposición, el enfoque histórico, asociado a la discusión sobre Ciencia, permite entender su carácter de construcción humana, es decir, sujeta al contexto socio-cultural en que está enmarcada.

Sin embargo, en un simposio realizado en 1970, en el Massachussetts Institute of Technology, una de las objeciones a la aproximación histórica en la enseñanza de Ciencia concernía precisamente a esa conciencia: el temor de que el conocimiento histórico, al debilitar la convicción de los jóvenes en la Ciencia, minara su espíritu científico, porque se cree que dicha convicción es necesaria para que el aprendizaje tenga éxito.

Según Burstyn (1972, apud Matthews, 1994b), de un lado, mientras los estudiantes pensaban de forma "convergente", buscando la respuesta "correcta", la Historia de la Ciencia tendría como característica esencial la complejidad. ¿Sería realmente 'posible utilizar material histórico — caracterizado por su complejidad, amplitud y divergencia para enseñar a personas interesadas en obtener la respuesta correcta [...]? ¿No sería la Historia, de algún modo subversiva a los propósitos pedagógicos de la Física?".

Thomas Kuhn, entre otros, había sostenido anteriormente este punto de vista: él señalaba el hecho de que la enseñanza de Ciencia se basa, al contrario de otras áreas del conocimiento, no en obras originales sino en libros de texto escritos especialmente para estudiantes. Según él, esta "iniciación dogmática a una tradición establecida" se justificaría por el hecho de que "ninguna parte de la Ciencia ha progresado tanto, o tan rápidamente, antes de que fuera posible esa educación convergente [...]". Así, Kuhn legitimaba la educación dogmática en función de sus resultados - la producción de científicos creativos e innovadores, los cuales no podrían ser innovadores sino tras una inmersión profunda en las concepciones ortodoxas de su disciplina, para sentirse parte de una tradición exitosa en la búsqueda de la verdad (1970, apud Matthews, 1994b, p. 76).

Uno de los puntos que se puede cuestionar en esa argumentación concierne a cuestiones educativas muy serias —como la adoctrinación- subyacentes en el hecho de cambiar la verdad histórica por el compromiso de los estudiantes hacia la empresa científica; por tanto se planteó reafirmar en las clases la idea de que la Ciencia estaría directamente asociada a la naturaleza, y los científicos sólo tendrían que extraerla o de velarla. En este sentido el discurso científico es ideológico, en la medida en que enfoca la Ciencia como actividad que se desarrolla en "torres de marfil", olvidando las complejas relaciones entre Ciencia-Tecnología-Sociedad (Moraes et al., 1990).

En contrapartida, es interesante el parangón que establece Michel Polanyi (apud Matthews, 1994a) con otras áreas del conocimiento. A él le parece obvio que la Historia y la Filosofía de la Ciencia deban por lo menos formar parte de la enseñanza de Ciencia, como la crítica literaria y musical forman parte la enseñanza literaria y musical. Sería muy raro pensar en un buen curso de Literatura que no incluyese elementos de crítica literaria, de historia de las formas literarias, el modo como la Literatura está subordinada, entre otros factores, a los períodos históricos y a los acontecimientos sociales. Sin embargo, es menos consensual la importancia de la comprensión histórica en los productos y métodos de la Ciencia.

Al demostrar que la Ciencia está subordinada a los contextos histórico y cultural vigentes en los diferentes momentos, la Historia y la Filosofía de la Ciencia contrarrestan 
concepciones equivocadas de la actividad científica, y sugieren, entre otros puntos, que el conocimiento científico actual es susceptible de transformación.

En las orientaciones provenientes de la Didáctica de las Ciencias la relativización del contenido también es un tema polémico. Las estrategias de cambio conceptual proponen sustituir las ideas del sentido común por ideas científicas. En contrapartida, otros autores sostienen que esta postura desconsidera el papel del conocimiento (Cobern, 1996), y del lenguaje a él asociado (Mortimer, 1996), en la vida cotidiana. Así defienden que, "en la nueva cultura del aprendizaje no se trata tanto de adquirir conocimientos verdaderos absolutos, ya dados -al fin de cuentas son pocos los que quedan- sino más bien de relativizar e integrar los sabores divididos" (Pozo, 1996).

Desde el punto de vista metodológico, una postura coherente no puede suponer, como lo advierte Joseph Schwab (apud Matthews, 1994b), que la Historia y Filosofía de la Ciencia se enseñen como "retórica de conclusiones". Por tanto, los aspectos metodológicos merecen atención y se analizarán a continuación.

\subsection{Sobre la forma}

Diversos estudios (apud Gil-Pérez, 1995) han aclarado las ideas de los profesores en lo que se refiere a la metodología científica, y señalan dos de sus características:

- Extremo inductivismo - no se considera la observación y la experimentación como sujetas a ideas apriorísticas, omitiendo el papel de las hipótesis y teorías.

- Visión rígida - el "Método Científico" se enfoca desde una perspectiva algorítmica, exacta, infalible, dogmática, y se considera como una secuencia lineal de etapas a ser cumplida paso a paso.

De esa forma, el sentido común subraya el tratamiento cuantitativo y el control, y olvida -incluso rechaza - cualquier relación con la creatividad, la inventiva y las tentativas preliminares.

En contraposición, la Historia y Filosofía de la Ciencia son necesarias para comprender la naturaleza de la actividad científica, evidenciando la dinámica del proceso de construcción del conocimiento. Así, cuestiones epistemológicas que podrían formularse en las clases de Ciencia serían, por ejemplo, la importancia de las creencias religiosas de los científicos, los aspectos metafísicos de los grandes debates, además de los factores políticos, económicos y sociales del desarrollo científico (Matthews, 1 994b).

La Historia y Filosofía de la Ciencia pueden presentar a los estudiantes los métodos de la investigación científica, en contraposición con la definición positivista del "método científico" presente en los libros didácticos. Laudan (1977), por ejemplo, plantea la subordinación de las teorías y tradiciones de investigación a una red más amplia de creencias y concepciones. Las respuestas de diferentes líneas de estudio a cuestiones tales como: ¿qué se puede reconocer como un problema relevante? ¿Cuáles objeciones se pueden considerar como problemas conceptuales? ¿Cuáles serian los criterios de inteligibilidad? ¿Cuáles los modelos de control experimental? -han cambiado drásticamente a lo largo de la historia y resultan distintas para culturas diferentes.

Por lo tanto, a los profesores habría que darles la oportunidad y los subsidios para abordar, en los cursos de Ciencia, puntos tales como: la variedad de interpretaciones 
racionales y plausibles que se pueden presentar para un mismo conjunto de datos y la distinción entre ecuaciones matemáticas, modelos y su interpretación física.

Pero el trabajo en el aula tiene que ser congruente con ese enfoque. Una alternativa es su aproximación al trabajo que desarrollan los científicos - presente, por ejemplo, en la enseñanza por investigación, donde la actividad en clase se modela a partir de las prácticas científicas (Duschl, 1995).

Gómez-Granell \& Coll (1994) señalan el carácter socio-cultural y contextual de la construcción del conocimiento, como Vygotsky lo había indicado antes. Considerando tal dimensión como característica no sólo de la construcción individual del conocimiento, sino también de la construcción del conocimiento científico, ésta resulta ser una de las características de la analogía entre actividad de investigación y el trabajo de los estudiantes en el aula, cuando desarrollan actividades de investigación.

En contrapartida, la educación científica no ha contribuido al contraste y al debate de ideas. Así, al igual que los medios masivos, "evita la complejidad, ofreciendo productos con interpretación literal, mejor dicho, mínima [...] La semiformación es característica de la comunicación masiva [...] [El semiculto] es autorreferido, aprende con el espacio y el tiempo, no reconoce diferenciaciones" (Mates, 1996, apud Vannucchi, 1997).

Sobresale, por ende, una de las características de la enseñanza por investigación: las situaciones de diálogo, que aportan un status muy diverso a las situaciones de conflicto cognitivo: no plantean a los alumnos el cuestionamiento externo de ideas personales, sino un trabajo de profundización, en donde ciertas ideas (tomadas como hipótesis) se sustituyen por otras (tan personales como las anteriores). No se trata de eliminar los conflictos cognitivos, sino de evitar que adquieran carácter de confrontación entre ideas propias (incorrectas) y los conocimientos científicos (correctos) (Carvalho \& Gil-Pérez, 1995).

Con una salvedad: estrategias pedagógicas acordes con la visión del conocimiento científico como conjunto de ideas indisociables de un contexto, no pueden tener el objetivo de eliminar determinadas ideas en función de otras — caso de las estrategias de cambio conceptual. Al fin y al cabo, ambas constituyen conocimientos válidos- cada cual en su contexto.

Coherentemente, la Historia y Filosofía de la Ciencia no pueden presentarse a los estudiantes como una "retórica de conclusiones". Como alternativa se propone presentarlas análogamente al contenido declarativo (hechos, términos y conceptos) (Driver, 1986; Duschl, 1995; Gil-Pérez, 1995; Wheatley, 1991), como situaciones problemáticas (Vannucchi, 1997).

Y, para que su discusión en el aula sea factible, se propone organizar la clase en grupos cooperativos, para que resulte innecesario que el profesor identifique un punto único a través del cual pueda coordinar el trabajo de todos los estudiantes concomitantemente (Carvalho et al., 1995), además de favorecer el desarrollo de habilidades de argumentación, pensamiento critico y la comprensión de los temas sometidos a discusión (Doise \& Mugny, 1984; Haste, 1987; Piaget \& Inhelder, 1969, apud Wheatley, 1991; Newman, Griffin \& Cole, 1989; Resnick, 1987; Rogoff \& Lave, 1984, apud Duschl, 1995).

Se señala también el papel fundamental del profesor a lo largo de la actividad (Coll, 1996; Gil-Pérez \& Carrascosa-Alis. 1994), en el sentido de fomentar la cooperación entre los 
alumnos, de aportar nuevas informaciones, de actuar como instigador, coordinador y para limitar los grados de libertad que los alumnos deban manejar (Mortimer \& Carvalho, 1996).

\section{LA ACTIVIDAD}

Se ha elaborado una actividad con la intención de observar cómo los estudiantes discuten sobre Ciencia cuando se les plantea un tema controvertido; en este caso, las relaciones entre Ciencia y Tecnología, con base en el episodio relativo al perfeccionamiento de la luneta por Galileo Galilei, en el siglo XVII.

La actividad se compone de un texto y algunas cuestiones formuladas a partir de los trabajos de historiadores, filósofos y sociólogos de la Ciencia de los cuales no se obtiene ningún consenso, ya sea entre modelos que establecen relación entre Ciencia y Tecnología, ya sea en la interpretación del episodio en cuestión por los historiadores de la Ciencia.

Así, por una parte, mientras el sentido común atribuye una relación causal entre desarrollo científico y tecnológico, considerando a la Ciencia como matriz de la Tecnología (Díaz, 1995), en el episodio de la luneta ese modelo no sólo no se aplica, sino que trata exactamente de lo contrario: aunque Galileo haya perfeccionado la luneta hasta el punto de permitir que se realizaran observaciones astronómicas — hecho que determinó una nueva etapa para la Astronomía-, la Ciencia de la época no explicaba por qué y cómo funcionaba aquel aparato.

Sólo en el año siguiente al episodio, Johannes Kepler publicaría Dioptrice, en el cual dedujo los principios del funcionamiento del telescopio analizando geométricamente la refracción de la luz a través de las lentes (Koestler, 1989).

Pero la formulación correcta de la ley de la refracción, asociada a un modelo explicativo, todavía no estaba establecida. Aunque Descartes y Snell la hubieran formulado de manera exacta, el primero, por considerar el rayo luminoso como una proyección de esferas - que pierden más velocidad al chocar con un cuerpo elástico que con un cuerpo duro-, había llegado a la concepción equivocada de que en un medio más denso, la velocidad de propagación de la luz aumentaría. Así, elaboró su ley correcta a partir de una hipótesis falsa, de un modelo inadecuado (Sabra, 1981). La demostración de Snell, a su vez, prescinde de un modelo explicativo, y se basa esencialmente en observaciones empíricas (Schurmann, 1946).

Los hechos sólo se aclararían unos 70 años más tarde, cuando Christian Huygens dedujo la ley de la refracción a partir del modelo de las ondas secundarias (Sabra, 1981).

Así, aunque Galileo haya transformado la "luneta débil en poderoso instrumento de estudio", lo hizo por haber sido el primero en pulir lentes objetivas de largo alcance con calidad suficientemente buena (Cohen, 1992); esto indica que si se establece una relación causal para este episodio, el instrumento tecnológico habrá permitido nuevas posibilidades a la Ciencia misma.

En contrapartida, sobre la influencia del microscopio en la Ciencia del siglo XVII —aparato contemporáneo a la luneta-, Pasteur afirmó, en 1864, que gracias a dicho descubrimiento la teoría de la generación espontánea, que estaba en decadencia, había tomado nuevo impulso (Gibert, 1982). 
Se nota que los ejemplos citados no aportan datos que se puedan encajar en un modelo sencillo. Mayr (1982) plantea el problema en los siguientes términos: se trata de datos empíricos que, en un gráfico, no arrojan puntos por los cuales se pueda trazar una curva suave. De esa forma, se comprueba la falsedad de la presumida invariabilidad histórica de la relación entre Ciencia y Tecnología. Todas las concepciones o modelos de esta relación tendrán limitaciones, y serán una tentación permanente en el sentido de hacer inferencias falsas, generalizaciones inconsistentes (Barnes \& Edge, 1982).

La interacción entre Ciencia y Tecnología estaría más bien relacionada con circunstancias hasta cierto punto aleatorias (personales, sociales, políticas y económicas) que con características permanentes de dichas áreas del saber. Price (1975) utiliza simbiosis, o sea, dependencia mutua y vital, como metáfora para la interacción.

En cuanto a la interpretación que hicieron del episodio historiadores de la Ciencia tales como Alexandre Koyré y Stillman Drake, Mac Lachlan (1990, apud Matthews, 1994a), comentarista de los trabajos comparados de estos dos autores, atribuye la diferencia en sus conclusiones, sobre todo a las distintas posiciones filosóficas. Así, el Galileo de Koyré parece habitar un mundo filosófico copernicano, platónico, de racionalismo y experimentos mentales. A los ojos de Drake, Galileo adquiere un carácter menos contemplativo y más activo - un agudo observador, experimentador e inventor.

Al preparar la actividad de enseñanza se seleccionó un diálogo escrito por Stillman Drake (1983), entablado por contemporáneos imaginarios de Galileo sobre el episodio de perfeccionamiento de la luneta. Un fragmento del texto presentado a los estudiantes sugiere que las primeras observaciones astronómicas realizadas por Galileo habrían ocurrido de casualidad:

"Sagredo [...] ¿Qué le hizo destinar un instrumento comercial y naval a los propósitos de la Astronomía?

Sarpi El folleto decía, al final, que estrellas invisibles alojo humano se podían observar a través de la luneta. Quizás nuestro amigo haya logrado comprobar ese hecho o tal vez lo haya descubierto por sí mismo. Ej.

Salviati [...] mientras probaba [el telescopio al atardecer, se le ocurrió apuntarlo hacia la Luna, creciente en ese momento. A través del telescopio la Luna se le presentó tan diferente de lo esperado, tanto con relación a su porción iluminada, como a la obscura, que durante todo el mes ocupó la atención exclusiva de nuestro amigo".

En clase, el carácter intencional de las observaciones astronómicas, punto controvertido para los historiadores, se volvió una cuestión polémica planteada por los alumnos: ¿Sería realmente de casualidad que Galileo giró el telescopio hacia la Luna? Lo que vio ¿era coincidentemente contrario a las expectativas de la teoría celeste aristotélica?

Así, las actividades de la clase se pueden elaborar de modo tal que "estimulen a los estudiantes a ejercitar la razón, y también a ser razonables" Los profesores deberían tratar de interesarlos en cuestiones filosóficas e históricas que puedan aflorar desde un tópico específico, en lugar de darles las respuestas definitivas, o imponerles sus propios puntos de vista" (Matthews, 1 994b).

De cualquier forma, ¿qué visión de Ciencia, de científicos y de conocimiento científico habría que presentarles a los estudiantes, ya que no hay una naturaleza de la Ciencia 
preferida ni siquiera por los filósofos de la Ciencia (Lederman, 1992, apud Alters, 1995) ${ }^{1}$. Lo que se recomienda es una enseñanza filosóficamente plural, es decir, que los estudiantes tengan noción de que existen múltiples interpretaciones para la Ciencia.

\section{EL AULA}

La actividad se propuso para clases de segundo curso secundario, (lo que correspondería en España al 20. de BUP o 4०. de ESO) en escuelas públicas de São Paulo. Brasil. Se filmaron las clases en video y luego se transcribieron. Los datos que se presentan a continuación corresponden a un episodio extraído de ellas.

El episodio, dividido en momentos, es corto si se lo compara con la duración de la clase. Sin embargo constituye un recorte cuya característica principal es pertenecer a un ciclo completo en el proceso de interacción entre los individuos, mediado por el objeto de conocimiento (Carvalho et al., 1992).

La selección e interpretación de los episodios están sujetas, desde luego, a los presupuestos teóricos del investigador. Justamente por eso, su enfoque refleja los aspectos que se buscan realzar y analizar: en este caso, la necesidad y el potencial de temas controvertidos para la educación científica.

En los tres momentos que se describen a continuación, en la primera clase los alumnos en grupos de 4 - 5 personas eyeron el texto y discutieron las cuestiones colocadas al final, Las frases transcritas, presentadas más abajo, corresponden a la clase siguiente, cuando el profesor planteó la discusión con todos los grupos simultáneamente.

\subsection{De la necesidad}

\section{MOMENTO 1}

En esta clase, tras la discusión, el profesor trata de sistematizar algunas conclusiones. Sin embargo, parte de los alumnos divergen de su posición, y eso lo lleva a introducir algunas ideas acerca del carácter de construcción permanente del conocimiento.

P ¿Estamos? Bueno, yo creo que la conclusión más importante en el episodio de la luneta es que la Tecnología precede la Ciencia. Así, esa idea de que la Ciencia genera Tecnología, resulta cuestionable, porque eso no siempre es verdad. En algunos casos puede ser, pero en otros no. Claro que ellas andan siempre lado a lado, en algunos momentos es fácil separarlas, en otros no.

\section{MO Para decir la verdad, no me convence.}

P Bueno, de eso se trata. Conocimiento es eso, conocimiento no es, tú no puedes, yo no te estoy midiendo la verdad. Estoy tratando de convencerte, ¿no es cierto?

\footnotetext{
${ }^{1}$ Esa falta de consenso entre los filósofos se puede ilustrar con el resutado encontrado en estudio realizado por Alters (1995) con 176 miembros de La Philosophy of Science Association (Y.U.LA.) a partir de definiciones de apriorismo, convencionalismo, positivismo y realismo. Además de haber adeptos para las cuatro categorías, otras 3 a 9 posiciones filosóficas afloraron de las respuestas.

*Equivalente al séptimo grado de Educación Básica en el caso colombiano. (N.E.)
} 
Dentro de algún tiempo tal vez lo puedas aceptar; o no, pero eso es el conocimiento, no es ... yo no vendo verdades. Conocimiento no es eso de las verdades, conocimiento científico no es la verdad absoluta, acabada. Si fuera la verdad absoluta, acabada, seria religión. Nosotros aquí no estamos haciendo religión. Estamos ha ciendo conocimiento, estamos construyendo conocimiento. $Y$ es eso. Pero tú puedes poner en cuestión lo que yo pienso.

CA Pero está probado que es verdad lo que dijiste, ¿no? O sea ... todo el mundo está de acuerdo que en ese caso realmente la Tecnología

P Mira...

CA La mayoría...?

P En realidad, cuando lees o haces Ciencia, siempre tienes un ala que dice que sí, y un ala que dice que no. Nunca hay un consenso colectivo de todos los historiadores, filósofos, que crean que Galileo fue eso. E incluso sobre Galileo hay mucha controversia, hay gente que cree que no, hay gente que cree que sí Por eso, cuando lees un texto debes citar la fuente [...]

GE ¿Está probado, profesor?

P ¿El qué?

GE En ese texto, ¿que su problema era tecnológico?

CA Nosotros no estamos de acuerdo.

P Bueno, pues de eso se trata: de discrepar.

KA ¿Y puedo quedarme con lo tecnológico y lo científico, entonces?

Las observaciones de las alumnas parecen indicar la necesidad de que las ideas se presenten como verdades (CA: Pero está probado que es verdad lo que dijiste, ¿no?; GE: ¿Está probado, profesor?).

Y la educación escolar ha apoyado esa postura, porque el ignorar las dimensiones histórica y filosófica de la Ciencia favorece una visión distorsionada de la actividad científica, basada en concepciones empiricoinductivistas: la Ciencia [y demás contenidos escolares, incluso la Historia misma] está compuesta de verdades incontestables. La rigidez e intolerancia de esta perspectiva subestima la creatividad del trabajo científico y crea un obstáculo intransponible para la enseñanza de Ciencia, pues, además de pretenciosa y reduccionista, capaz de atribuirle a la Ciencia características inapropiadas, tal perspectiva acaba por moldear el comportamiento del estudiante a esa imagen - el pensamiento divergente y las opiniones conflictivas no se consideran importantes, incluso a veces, se consideran negativos (Gil-Pérez, 1985, apud Castro \& Carvalho, 1995).

Por eso es importante que los estudiantes vivan situaciones de conflicto de ideas, lo cual puede contribuir a hacerlos reflexionar sobre el status negativo que tales situaciones conllevan. Fue lo que ocurrió en esta clase: como los estudiantes habían reflexionado y discutido previamente en sus grupos, se sintieron seguros al defender un punto de vista 
contrario al del profesor, presentando sus argumentos como corresponde, hecho que se observa en el Momento2.

\subsection{Del Potencial}

\section{MOMENTO 2}

Al discutir con el profesor y con los demás alumnos de la clase sobre la naturaleza de las dificultades que tuvo Galileo al construir la luneta, algunos grupos defendieron su carácter tecnológico y otros, su carácter científico.

El profesor hizo su síntesis:

P Vamos a ver: el problema que encontró Galileo fue un problema de carácter tecnológico, técnico. El tenía que pulir los lentes, a lo mejor sin saber por qué los lentes tenían esas propiedades. Galileo no sabía, nadie sabia en esa época, explicar por qué los lentes funcionaban así ¿correcto? Y ahí uno puede distinguir muy bien qué es técnica y qué es Ciencia. Porque la Ciencia es, ella exige que uno conozca la explicación de las causas, del porqué. Si Galileo hubiera hecho Ciencia en el caso del episodio del telescopio, él sabría, o tendría que saber explicar cómo y por qué funcionaban los lentes, pero ni él ni nadie lo sabía en esa época. Pero resulta que aún sin tener ese conocimiento, perfeccionó el instrumento, pulió los lentes y obtuvo resultados cada vez mejores. Por lo tanto el problema que tuvo Galileo fue un problema tecnológico, no científico. ¿Estamos?

CA ¿Pero la falta de conocimiento no es un problema científico? No podía saber hacerlo, no era un ... no había profundizado el conocimiento científico como para hacer aquello ¿no?

P Pero es un problema técnico. El debía tener un instrumento para pulir el lente, que era un problema mucho más práctico, mucho más técnico que saber explicar las causas y los porqués. El problema cien tífico, en ese caso, es saber explicar por qué los lentes aumentan los objetos de tamaño. A él no le interesaba contestar esta pregunta.

MA Sólo que, por ejemplo, si él hubiera tenido el conocimiento científico de los lentes, cuando los iba a hacer por primera vez, ya los habría hecho cóncavos

P Justamente. Esa es una cuestión importante: ¿que es conocimiento científico? Porque si el tuviera ese conocimiento científico, podría proveer; sabría anticipar el resultado. Pero el no lo sabía, ¿de acuerdo? Es porque el conocimiento científico abarca además de una explicación, el prever [...]

GE Pero desde el momento en que se puso a intentarlo y llego a la conclusión de que si dejaba el lente curvo obtendría efecto, eso ya seria conocimiento científico.

P No seria conocimiento científico porque el no sabía explicar por que el lente curvo iba a producir aquel resultado. ¿Por que el lente plano no hacia nada y el lente curvo producía aquel efecto? El sabia, por sus observaciones, que el lente curvo tenía un resultado mejor que el lente plano (que no producía resultado alguno). Esto es una observacion, ¿correcto? ¿Por que? El no sabía contestar. 
AN Pero no es solo el aspecto tecnológico. Yo creo que ahí están los dos relacionados. Tanto el tecnológico, como el científico. En este caso no hay cómo distinguir si es uno de los dos.

Inicialmente el profesor planteo su punte de vista, pero los alumnos no estaban convencidos. CA señaló un aspecto pertinente: Galileo encaré, como problema, la falta de conocimiento científico (Pero la falta de conocimiento no es un problema científico?). Sin embargo, al parecer ella no alcanzó a reconocer que ese desconocimiento no representó un obstáculo para lograr el perfeccionamiento de la luneta (No podía saber hacerlo, no era un ... no había pro fundízado el conocimiento científico como para hacer aquello ¿no?).

Se pueden plantear dos hipótesis: en primer lugar, una confusión entre saber y, usando sus propias palabras, saber hacer" Otra interpretación es que a! concebir una relación causal Ciencia ${ }^{\circledR}$ Tecnología, CA razono de manera análoga a Bacon: siendo la causa ignorada. se frustra e! efecto" (1973. aforismo III, libro 1).

Insatisfechos con la explicación del profesor, los alumnos señalaron puntos importantes acerca de que es la actividad científica, tales como la previsión (MA: sí el hubiera tenido el conocimiento científico de los tontos, cuando los hizo por primera vez, ya los habría hecho cóncavos ...) y la descripción (GE: Pero desde el momento en que se puso a intentarlo y llego a la conclusión de que sí dejaba el lente curvo obtendría efecto, eso sería conocimiento científico). $\mathrm{Y}$ a este segundo rasgo de la actividad científica -la descripción-, e! profesor contrapuso su concepción: Esto es una observación, ¿correcto? ¿ Y donde esta la explicación? No sería conocimiento científico porque él no sabía explicar el porqué [...]

Esto es lo más importante: que los alumnos revisen y ensanchen sus representaciones de Ciencia y Tecnología. La contraposición de ideas diferentes, además de relativizarlas y plantear la necesidad de justificar puntos de vista, puede conducir a la toma de conciencia y a aclarar ideas inicialmente indiferenciadas. Parafraseando a Siegel (1993) (que se refiere a la concepciónn de Ciencia), "deberíamos buscar para nuestros alumnos lo mismo que buscamos para nosotros: una conciencia y una apreciación cada vez mas profundas de los problemas y dudas de nuestra[s] concepción [ciones]".

\section{MOMENTO 3}

Como se podrá observar en el fragmento que sigue, la discusión acabo por desviarse del tema relaciones Ciencia-Tecnología para entrar, usando las palabras del profesor, "en el terreno de las intenciones” que Galileo habría tenido cuando perfecciono la luneta.

P Mire, el no tenía el conocimiento científico.

GE Así es. Le faltaba eso.

P Muy bien. En este sentido su problema era un problema científico. El no estaba preocupado en explicar el porqué. Estaba preocupado

DE ¡Es que no se sabe!

P ¿Cómo dices?

DE No hay cómo saber si él estaba... 
P Bueno, por lo menos ahí; históricamente. El estaba preocupado en perfeccionar el lente y en observar el resultado - sí el lente era capaz de aumentar el tamaño del objeto.

A lo largo de la discusión los alumnos tuvieron la oportunidad de plantear nuevas cuestiones más allá de lo propuesto. Así, al analizar el "terreno de las intenciones", los alumnos señalaron eventuales objetivos científicos de Galileo respecto a la luneta - no en el sentido de comprender su funcionamiento, sino de llevar a cabo observaciones celestes:

P Una pregunta que quisiera hacer ahora, que salió aquí en este grupo, es la siguiente: ¿Por qué Galileo apuntó El telescopio hacia la Luna? ¿Habrá sido de casualidad?

(Algunos alumnos dijeron que no.)

P ¿ ¿Será cierto?

MI Es que si no fue de casualidad, fue por conocimiento científico.

P Ahí está: ahora entramos al terreno de las intenciones. Es decir, Galileo era un genio, es muy posible. Además de ser consultor militar era también científico. Nosotros no podemos decir que fue así; tampoco podemos asegurar que si o no, él apuntó el telescopio hacia la Luna de casualidad, sin querer, la miró y dijo Mira, la Luna es asi”. ¿No será que él ya tenía una concepción del mundo, una teoría, un conocimiento que lo impulsé a...

DE Para mi que sí tenía...

P ... a apuntar el telescopio a la Luna? ¿ Será que tendría una intención previa?

(Una parte de la clase está de acuerdo.)

P ¿O fue de casualidad? Es muy difícil saberlo.

LI Quizás haya sido por mora curiosidad.

P Puede ser ... pero no hay cómo saberlo.

La duda de los alumnos acerca de las intenciones de Galileo constituye un punto controvertido entre Los mismos historiadores de la Ciencia, y hay dos argumentos que sostienen su relevancia: la imposibilidad de una versión final y correcta para todas las disputas entre puntos de vista diferentes, y la importancia pedagógica de los debates y contraposición de ideas.

Sin embargo, la inclusión de temas contradictorios entre los propios filósofos e historiadores de la Ciencia requiere que se vuelvan a plantear los objetivos educativos; en tal caso, no fomentar respuestas finales, sino '[...] algún insight sobre el modo como trabajan los científicos o cómo se obtiene el nuevo conocimiento científico" (Kipnis, 1995, p. 613). 


\section{DISCUSIÓN}

De acuerdo con lo anteriormente planteado, se entiende que contenido y metodología son cuestiones complementarias - lo cual ya es una realidad en relación con la metodología y contenido que tradicionalmente se imparten en los cursos de Ciencia, y debe serlo también respecto al enfoque histórico.

En una sistematización de propuestas constructivistas, una de las características que destaca Driver (1986) es que se tengan en cuenta los conocimientos e ideas previas de los estudiantes. De hecho, se entiende que las ideas de los alumnos se deben tener en cuenta tanto para la planificación didáctica, como en las situaciones de enseñanza. Además hay que estar atento a la necesidad de reestructurar dichas ideas.

Conviene detenerse en este punto: ¿de qué orden serían tales reestructuraciones? Contrario a las estrategias de cambio conceptual, se entiende que "el aprendizaje significativo [...] no es una cuestión del todo o nada" (Coll, 1996, p. 141).

Así por ejemplo, se sabe que los estudiantes ya tienen ideas respecto a lo que constituye el conocimiento científico, como también sobre sus relaciones con la Tecnología. Sin embargo, por lo que se refiere al contraste entre las informaciones planteadas en el texto de una actividad, y los aportes en Las discusiones con el profesor y demás alumnos, no se espera, necesariamente, que determinadas concepciones filosóficas cambien.

Se entiende La toma de conciencia por parte del estudiante, acerca de sus concepciones, como aspecto importante del proceso de enseñanza-aprendizaje. Esa conciencia, alentada por la revisión de ideas, puede impulsar hacia un cambio de concepción, hecho que se puede apreciar en la frase de una alumna de una de las clases en que se desarrollé la actividad:

P Tu respuesta ¿no "está de acuerdo sobre el desarrollo científico y tecnológico"?

AN No. No está de acuerdo con lo que nosotros pensábamos antes.

P Ah, antes. ¿Y por qué? ¿Qué pensabais antes?

AN Que la Ciencia venía antes de la Tecnología.

Por otra parte, aunque esto no suceda siempre, lo más importante es que las actividades en Historia y Filosofía de la Ciencia impulsan a los alumnos a revisar y ampliar sus representaciones, y así adquieren "conciencia y apreciación cada vez más profundas de los problemas y dudas" de éstas.

Esta posición no supone sonsacar las ideas de los alumnos para luego contrastarlas, al contrario, valorar las ideas de los alumnos, favoreciendo su creatividad y autonomía. De ese modo, el planteamiento que se defiende para la reestructuración de las, ideas de los estudiantes no considera, tan sólo, el conocimiento del sujeto, su historia pasada, sino también su futuro, sus perspectivas.

Hay que señalar, sin embargo, que no se trata de impulsar a los estudiantes a dar sus opiniones a despecho de su conocí-miento, actitud que desvalorizaría la adquisición escolar de conocimiento y el pensamiento fundado. Además de no contribuir al 
aprendizaje, la presentación de puntos de vista personales y sin fundamento consolida las prácticas referidas a si mismo.

En eso estriba la importancia del conocimiento histórico, que permite que Las discusiones filosóficas se hagan dentro de su contexto histórico, y aporten subsidios para debates fundados.

Se entiende que "aprender Ciencias [y aprender sobre Ciencias] requiere que los jóvenes accedan a una forma diferente de pensar y de explicar el mundo: volverse socializado en mayor o menor extensión, en las prácticas de la comunidad científica con sus propósitos particulares y sus maneras de ver y de explicar peculiares" (Driver et al., 1994, apud Mortimer \& Carvalho, 1996). Cuando están realmente involucrados en ese "proceso de aculturación", los estudiantes se disocian de las prácticas referidas a sí mismos, ya que para comprender esa nueva forma de ver el mundo, las ideas que traen no bastan o no son congruentes

Paralelamente, dentro de esta perspectiva, el desarrollo de habilidades cognitivas y argumentativas —una de las realizaciones más importantes de la educación científica (Deanna Kuhn, 1991, 1993, apud Duschl, 1995) — también resultan favorecidas, ya que los estudiantes deben justificar y debatir sus puntos de vista.

Ahí se sitúa la interface dlrecta entre eL contenido y el modo de introducir en clase la Historia y la Filosofía de la Ciencia. En este sentido, cuando los alumnos trabajan en grupo, cuando discuten sus ideas con los compañeros y con el profesor, se fomenta el desarrollo de habilidades de razonamiento, argumentación, expresión de ideas, además de la necesidad de reflexionar y respetar las ideas de los demás.

Otro punto por considerar, fundamental en cualquier propuesta didáctica, concierne al papel del profesor. En primer lugar, hay que resaltar su importancia. Aunque no se pueda ignorar la dinámica interna de construcción del conocimiento, y tampoco se la pueda reemplazar mediante la intervención pedagógica, tal intervención es importante y consiste esencialmente en crear las condiciones adecuadas para que la dinámica interna ocurra y vaya orientada en determinada dirección, de acuerdo con las intenciones educativas (Coll, 1996).

Cualesquiera que sean los medios, si las intenciones educativas enfocan las verdades, cuando el profesor, con la postura típica de esa orientación, se dirige a los alumnos con una pregunta que no es pregunta — “¿Tenéis alguna duda?”-, tendrá grandes posibilidades de recibir como respuesta una pregunta: “¿Cuál es la fórmula?"

\section{REFERENCIAS BIBLIOGRÁFICAS}

Alters, B. J. (1995). Whose Nature of Science? En F. Finley, D. Allchin, D. Rhees, S. Fifield (eds.), Third International History, Philosophy, and Science Teaching Conference, Minneapolis, pp. 33-47.

Bacon,F, (1973). Novum Organum Verdadeiras Indicaçoes Acerca da Interpretação da Natureza. J. A. R. de Andrade (trad.). São Paulo: Abril Cultural.

Barnes, B.; Edge, D. (1982). Science in Context-Readings in the Sociology of Science. London: The Open University Press. 
Carvalho, A. M. P. de (1989). Formação de professores: o discurso critico-liberal em oposíção ao agir dogmático repressivo. Cíencia e Cultura, vol 41, No 5, pp. 432-434.

Carvalho, A. M. P. de: Garrido, E.; Castro, R. S. (1995). El pape] de las actividades en la construcción de: conocimiento en clase". Investigación en la Escuela, No 25, pp. 61-70.

Carvalho, A. M. P. de; Castro, R. S.; Laburu, C. E.; Mortimer, E. F. (1992). Pressupostos epistemológicos para a pesquisa em ensino de ciencias". Cadernos de Pesquisa, no 82, pp. 85-89.

Carvalho, A. M. P. de; Gil-Pérez, D. (1995). Formação de Professores de Ciências. 5. Valenzuela (trad.). São Paulo: Cortez.

Castro, RS.; Carvalno, A. M. P. de (1995). The Historic Approach ín Teaching: Analysis of an Experience. Science \& Education, vol, 4, no 1, pp. 65-85.

Cobero, W. W. (1996). Worldview Theory and Conceptual Chango in Science Education, Science Education, vol. 80, no 5, pp. 579-610.

Cohen, B. I. (1992). The Birth of a New Physics. London: Penguin Books.

Coll, C. (1996). Psicología e Currículo - Uma aproximação psicopedagógica á elaboração do currículo escolar. São Paulo: Atica.

Drake, S. (1983). Telescopes, Tides and Tactícs - A Galilean Dialogue about the Starry Messenger and Systems of the World. Chicago: The University of Chicago Press.

Driver, R. (1986). Psicología Cognoscitiva y Esquemas Conceptuales de los Alumnos. Enseñanza de las Ciencias, vol. 4, no 1, pp. 3-15.

Duschl, R. A. (1995). Más allá del conocimiento: los desafíos epistemológicos y sociales de La enseñanza mediante el cambio conceptual. Enseñanza de las Ciencias, vol. 13, no 1, pp. 3-14.

Gibert, A. (1982). Origens Históricas da Física Moderna. Lisboa: Fundação Calouste Gulbenkian.

Gil-Pérez, D. (1993). Contribución de la historia y de la filosofía de las ciencias al desarrollo de un modelo de enseñanza/aprendizaje como investigación. Enseñanza de las Ciencias, vol. 11, no. 2, pp. 197-212.

Gil-Pérez, D. (1995). New trends in science education. International Journal of Science Education, (preprint).

Gil- Pérez, D.; Carrascosa-Alis, J. (1994). Bringing Pupils' Learning Closer to a Scientific Construction of Knowledge: A Permanent Feature in innovations in Science Teaching. Science Education, vol. 78, no. 3, pp. 301-315.

Gómez-Granell, C.; COLL, C. (1994). ¿De qué hablamos cuando hablamos de constructivismo? Cuadernos de Pedagogía, 221, pp. 8-10. 
Kipnis, N. (1995). Blending Physics with History. En F. Finley, D. Allchin, D. Rhees, S. Fifield (eds.).Third International History, Phílosophy, and Science Teaching Conference. Minneapolis.

Koestler, A. (1989). O Homem e Universo (The Sleepwalkers - The History of Man's Changing Vision of the Universe). A. Denis (trad.). São Paulo: Ibrasa.

Laudan, L. (1977). Progress and Its Problems - Towards a Theory of Scientific Growth. Berkeley: University of California Press.

Matthews, M. R. (1 994a). Historia, filosofía y enseñanza de las ciencias: la aproximación actual. Enseñanza de las Ciencias, vol. 12, no 2, pp. 255-277.

Matthews, M. R. (1994b). Science Teaching- The Role of History and Philosophy of Science. New York: Routledge.

Mayr, O. (1982). The science-technology relationship. En B. Barnes, D. Edge (eds.), Scíence in Context - Readings in the Sociology of Science. London: The Open University Press.

Moraes, A. G. et al. (1990). Representações sobre Ciência e suas ímplicações para o Ensino de Fisica. Caderno Catarinense de Ensino de Física, vol. 7, no 2, pp. 115-122.

Mortimer. E. F. (1996). Construtívismo, mudança conceitual e ensíno de ciências: para onde vamos 7 Investigações em Ensino de Ciências, vol. 1, no 1, pp. 20-39.

Mortimer, E. F.; Carvalho, A. M. P. de (1996). Referenciais teóricos para análise do processo de ensino de Ciências. Cadernos de Pesquisa, no 96, pp. 5-14.

Pozo, J. I. (1996). Aprendices y maestros - la nueva cultura del aprendizaje. Madrid: Alianza Editorial.

Sabra, A. I. (1981). Theories of Light - from Descartes to Newton. Cambridge: Cambridge University Press.

Schurmann, P. F. (1946). Luz y Calor - 25 siglos de hipótesis acerca de su naturaleza. Buenos Aires: Espasa-Calpe Argentina.

Siegel, H. (1993). Naturalized Philosophy of Science and Natural Science Education. Science \& Education, vol. 2, no 1, pp. 57-68.

Trumbull, D. J. (1987). The Irrelevance of Cognitive Science to Pedagogy: Absence of a Context. En H. Hugh, J. Novak (eds.), Second International Seminar Misconceptions in Science and Mathematics, Ithaca, pp. 490- 495.

Vannucchi, A. I. (1997). História e Filosofía da Ciência - da teoría para a sala de aula. Dissertação de Mestrado apresentada ao Instituto de Física e à Faculdade de Educação da Uníversidade de Sáo Paulo, SSo Paulo.

Wheatley, G. H. (1991). Constructivist Perspectives on Science and Mathematics Learning. Science Education, vol. 75. no 1. pp. 9-21. 